\title{
Convergence Evaluation of a Load Flow Method based on Cespedes' Approach to Distribution System Analysis
}

\author{
Diego Issicaba ${ }^{1}$ and Jorge Coelho ${ }^{2}$ \\ ${ }^{1}$ Department of Electrical Engineering, Federal University of Technology - Parana (UTFPR), Curitiba-PR, Brazil \\ ${ }^{2}$ Department of Electrical Engineering, Federal University of Santa Catarina (UFSC), Florianopolis-SC, Brazil
}

\begin{tabular}{|c|c|}
\hline Article Info & ABSTRACT \\
\hline $\begin{array}{l}\text { Article history: } \\
\text { Received Aug 9, } 2016 \\
\text { Revised Sep 20, } 2016 \\
\text { Accepted Oct 7, } 2016\end{array}$ & $\begin{array}{l}\text { This paper evaluates the convergence of a load flow method based on Cespedes' for- } \\
\text { mulation to distribution system steady-state analysis. The method is described and the } \\
\text { closed-form of its convergence rate is deduced. Furthermore, convergence dependence } \\
\text { of loading and the consequences of choosing particular initial estimates are verified } \\
\text { mathematically. All mathematical results have been tested in numerical simulations, }\end{array}$ \\
\hline
\end{tabular}

Power engineering

Power distribution systems

Load flow analysis

Convergence

Copyright (c) 2016 Institute of Advanced Engineering and Science. All rights reserved.

\section{Corresponding Author:}

Diego Issicaba

Department of Electrical Engineering, Federal University of Technology - Parana (UTFPR)

Av. Sete de Setembro, 3165, Sector D, Rebouças, 80230-910 Curitiba-PR, Brazil

+55 $413310-4626$

issicaba@utfpr.edu.br

\section{INTRODUCTION}

Load flow methods are fundamental tools to power distribution system analysis $[1,2]$. These methods allow computing steady-state voltages at network nodes as well as the amount of power flowing through power system devices. Nevertheless, power system literature lacks on formal analysis and comparisons among convergence properties of load flow algorithms. Consistent exceptions can be found in $[3,4,5,6,7]$. Particularly, in [6] the convergence of a forward-backward sweep method is evaluated and its dependence on system loading verified. In [7], this same method is formally assessed using fixed-point concepts and the contraction mapping theorem.

In this context, this paper introduces the convergence analysis of a load flow method based on $\mathrm{R}$. G. Cespedes' approach to distribution system analysis [8]. For this accomplishment, section 2 introduces the proposed method focusing on algorithm procedures and main equations. Section 3 presents the deduction of the convergence rate of the algorithm and a mathematical region where algorithm iterates are confined, as long as initial estimates are chosen properly. In section 4, numerical results are shown to illustrate the validity of the mathematical developments. At last, section 5 outlines conclusions and final remarks.

\section{A LOAD METHOD INSPIRED ON CESPEDES' FORMULATION}

Consider the radial feeder schematic shown in Fig. 1. In the schematic, $z_{i}$ denotes a series line impedance, $E_{i}=e_{i}+j f_{i}$ represents complex node voltages, and $S_{L_{i}}$ denotes complex loads, all refereed to a general node $i$. The index $u_{i}$ stands for the node upstream node $\mathrm{i}$. The substation bus is named the 0 (zero) node with complex voltage denoted by $E_{0}$. Furthermore, the index $i$ points out to both a node and its upstream line.

The complex power flow downstream line $i$ can be computed by summing the loads and losses down- 


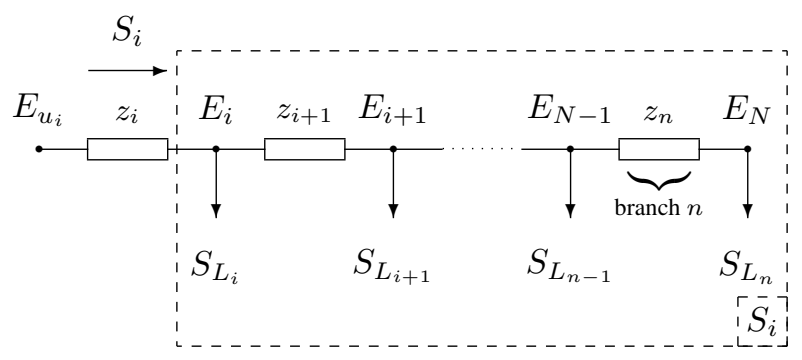

Figure 1. Radial distribution network schematic (adapted from [1]).

stream the line as follows

$$
S_{i}=S_{L_{i}}+\sum_{r \in \Delta_{i}} S_{L_{r}}+\sum_{r \in \Delta_{i}} z_{r}\left|\frac{S_{r}^{*}}{E_{r}^{*}}\right|^{2}
$$

where $\Delta_{i}$ represents either the set of nodes downstream node $i$ or downstream line $i$, depending on the variables involved. As consequence, $S_{i}$ can be expressed as function of variables associated to nodes immediately downstream line $i$, as follows

$$
S_{i}=S_{L_{i}}+\sum_{d \in \mathcal{D}_{i}} S_{d}+\sum_{d \in \mathcal{D}_{i}} z_{d}\left|\frac{S_{d}^{*}}{E_{d}^{*}}\right|^{2}
$$

where $\mathcal{D}_{i}$ denotes either the set of nodes immediately downstream node $i$ or immediately downstream line $i$.

Furthermore, the difference between node voltages in adjacent nodes can be expressed as

$$
E_{i}=E_{u_{i}}-z_{i}\left(\frac{S_{i}^{*}}{E_{i}^{*}}\right)
$$

Separating the real and imaginary parts of (2) and the voltage magnitudes in (3), the recursive equations utilized in the load flow method proposed by R. G. Cespedes [8] can be deduced. Conversely, by applying (2) and (3) without further deductions, a similar but also effective load flow method can be designed. Starting from initial complex voltage estimates, the two steps below can be continually repeated until the convergence of complex voltages is reached.

1. In a backward process, the complex power flow at each node is calculated using (2), starting at end-nodes and stopping at the first node immediately downstream from the substation node.

2. In a forward process, complex voltage are updated, away from the substation node, using (3).

The complex voltage at the substation bus is assumed constant during the procedures. The convergence criterion refers to the maximum absolute mismatch between subsequent complex voltage iterates.

\section{CONVERGENCE ASSESSMENT}

This section addresses the converge assessment of the algorithm, focusing on aspects related to the implications of choosing an initial estimate and the deduction of the convergence rate.

\subsection{Initial Estimate}

The update rule of the algorithm can be written recursively for iteration $k$ as

$$
\psi_{i}^{(k)}=\psi_{u_{i}}^{(k)}-\frac{z_{i}}{E_{i}^{(k) *}}\left(S_{i, c}^{*}+L_{i, a c}^{(k) *}\right)
$$

where $E_{i}^{(k)}$ denotes the complex voltage at node $i$ and iteration $k, \psi_{i}^{(k)}$ is the complex voltage at node $i$ and iteration $k+1, S_{i, c}$ denotes the sum of all complex loads downstream node $i$ (including the one at node $i$ ) and $L_{i, a c}^{(k)}$ represents the sum of all electrical losses downstream node $i$ and iteration $k$. 
Let us define $\hbar_{i}$ as the set of lines in the path between node 0 and $i$. By (4), we have

$$
\begin{array}{ccccc}
\psi_{1}^{(k)} & = & E_{0} & - & \frac{z_{1}}{E_{1}^{(k) *}}\left(S_{1, c}^{*}+L_{1, a c}^{(k) *}\right) \\
\vdots & = & \psi_{1}^{(k)} & - & \vdots \\
\vdots & = & \vdots & - & \vdots \\
\psi_{i}^{(k)} & = & \psi_{u_{i}}^{(k)} & - & \frac{z_{i}}{E_{i}^{(k) *}}\left(S_{i, c}^{*}+L_{i, a c}^{(k) *}\right)
\end{array}
$$

By summing the equations above, a closed-form for the update rule of the algorithm can be obtained.

$$
\psi_{i}^{(k)}=E_{0}-\sum_{m \in \hbar_{i}} \frac{z_{m}}{E_{m}^{(k) *}}\left(S_{m, c}^{*}+L_{m, a c}^{(k) *}\right)
$$

For instance, consider a radial network with 5 nodes and connections $\{0-1,1-2,2-3,1-4\}$. For this network, the closed-form for the update rules are the following:

$$
\begin{aligned}
& \psi_{1}^{(k)}=E_{0}-\frac{z_{1}}{E_{1}^{(k) *}}\left(S_{1, c}^{*}+L_{1, a c}^{(k) *}\right) \\
& \psi_{2}^{(k)}=E_{0}-\frac{z_{1}}{E_{1}^{(k) *}}\left(S_{1, c}^{*}+L_{1, a c}^{(k) *}\right)-\frac{z_{2}}{E_{2}^{(k) *}}\left(S_{2, c}^{*}+L_{2, a c}^{(k) *}\right) \\
& \psi_{3}^{(k)}=E_{0}-\frac{z_{1}}{E_{1}^{(k) *}}\left(S_{1, c}^{*}+L_{1, a c}^{(k) *}\right)-\frac{z_{2}}{E_{2}^{(k) *}}\left(S_{2, c}^{*}+L_{2, a c}^{(k) *}\right)-\frac{z_{3}}{E_{3}^{(k) *}}\left(S_{3, c}^{*}+L_{3, a c}^{(k) *}\right) \\
& \psi_{4}^{(k)}=E_{0}-\frac{z_{1}}{E_{1}^{(k) *}}\left(S_{1, c}^{*}+L_{1, a c}^{(k) *}\right)-\frac{z_{4}}{E_{4}^{(k) *}}\left(S_{4, c}^{*}+L_{4, a c}^{(k) *}\right)
\end{aligned}
$$

and, in a compact matrix notation:

$$
\left[\begin{array}{c}
\psi_{1}^{(k)} \\
\psi_{2}^{(k)} \\
\psi_{3}^{(k)} \\
\psi_{4}^{(k)}
\end{array}\right]=\left[\begin{array}{c}
E_{0} \\
E_{0} \\
E_{0} \\
E_{0}
\end{array}\right]-\left[\begin{array}{cccc}
\frac{z_{1}}{E_{1}^{(k) *}} & 0 & 0 & 0 \\
\frac{z_{1}}{E_{1}^{(k) *}} & \frac{z_{2}}{E_{2}^{(k) *}} & 0 & 0 \\
\frac{z_{1}}{E_{1}^{(k) *}} & \frac{z_{2}}{E_{2}^{(k) *}} & \frac{z_{3}}{E_{3}^{(k) *}} & 0 \\
\frac{z_{1}}{E_{1}^{(k) *}} & 0 & 0 & \frac{z_{4}}{E_{4}^{(k) *}}
\end{array}\right]\left[\begin{array}{c}
S_{1, c}^{*}+L_{1, a c}^{(k) *} \\
S_{2, c}^{*}+L_{2, a c}^{(k) *} \\
S_{3, c}^{*}+L_{3, a c}^{(k) *} \\
S_{4, c}^{*}+L_{4, a c}^{(k) *}
\end{array}\right]
$$

This example suggests the update rule can be written in a general compact matrix notation. For this accomplishment, assuming an indexing where $u_{i}<i$, let us define the lower triangular path matrix $\mathbf{T}$ with size $n \times n$ and entries

$$
t_{i m}= \begin{cases}1, & \text { if } m \in \hbar_{i} \\ 0, & \text { otherwise }\end{cases}
$$

Then, the update rule of the algorithm can written in the compact form

$$
\mathbf{\Psi}^{(k)}=\mathbf{E}_{\mathbf{0}}-\mathbf{T} \mathbf{Z}_{\mathbf{p}} \mathbf{S}_{\mathbf{c}}^{*} \mathbf{K}^{(k) *}-\mathbf{T Z}_{\mathbf{p}} \mathbf{L}_{\mathbf{a c}}^{(k) *} \mathbf{K}^{(k) *}
$$

where $\Psi^{(k)}$ is a $n \times 1$ vector with entries given by $\psi_{i}^{(k)}, \mathbf{E}_{\mathbf{0}}$ is a $n \times 1$ vector with entries equal to $E_{0}, \mathbf{Z}_{\mathbf{p}}$ is the $n \times n$ primitive impedance matrix, $\mathbf{S}_{\mathbf{c}}$ denotes a $n \times n$ diagonal matrix with elements equal to $S_{i, c}, \mathbf{L}_{\mathbf{a c}}^{(k)}$ represents a $n \times n$ diagonal matrix with elements given by $L_{i, a c}^{(k)}$ and $\mathbf{K}^{(k)}$ is a $n \times 1$ vector with reciprocals of $E_{i}^{(k)}$.

Let now $\mathcal{R}$ be a closed region in the complex space $C^{n}$ defined by $\mathcal{R}=\left\{\mathbf{E} \in C^{n},\left\|E_{i}\right\| \geq\left(E_{0}-\alpha\right)\right.$, $\forall i=1, \ldots, n\}$, for some $\alpha \in R$ such that

$$
\frac{E_{0}}{2} \leq \alpha<E_{0}-\sqrt{\rho_{\alpha}}
$$

where

$$
\rho_{\alpha}=\left\|\mathbf{T} \mathbf{Z}_{\mathbf{p}} \mathbf{S}_{\mathbf{c}}^{*}\right\|+\left\|\mathbf{T} \mathbf{Z}_{\mathbf{p}}\right\|\left\|\mathbf{L}_{\mathbf{a c}, \alpha}^{*}\right\|
$$


and $\mathbf{L}_{\mathbf{a c}, \alpha}$ is a $n \times n$ diagonal with entries given by the maximum accumulated losses downstream each node, computed using a backward process with voltage magnitudes equal to $\left(E_{0}-\alpha\right)$.

Given an iterate $\mathbf{E}^{(k)} \in \mathcal{R}$, by (13) we have

$$
\begin{aligned}
\left\|\mathbf{\Psi}^{(k)}-\mathbf{E}_{\mathbf{0}}\right\| & =\left\|\mathbf{T} \mathbf{Z}_{\mathbf{p}} \mathbf{S}_{\mathbf{c}}^{*} \mathbf{K}^{(k) *}+\mathbf{T} \mathbf{Z}_{\mathbf{p}} \mathbf{L}_{\mathbf{a c}}^{(k) *} \mathbf{K}^{(k) *}\right\| \\
& \leq\left\|\mathbf{T} \mathbf{Z}_{\mathbf{p}} \mathbf{S}_{\mathbf{c}}^{*}\right\|\left\|\mathbf{K}^{(k) *}\right\|+\left\|\mathbf{T} \mathbf{Z}_{\mathbf{p}}\right\|\left\|\mathbf{L}_{\mathbf{a c}}^{(k) *}\right\|\left\|\mathbf{K}^{(k) *}\right\| \\
& \leq \frac{\left\|\mathbf{T} \mathbf{Z}_{\mathbf{p}} \mathbf{S}_{\mathbf{c}}^{*}\right\|}{\left(E_{0}-\alpha\right)}+\frac{\left\|\mathbf{T} \mathbf{Z}_{\mathbf{p}}\right\|\left\|\mathbf{L}_{\mathbf{a c}, \alpha}^{*}\right\|}{\left(E_{0}-\alpha\right)}
\end{aligned}
$$

and, by using (14) we have

$$
\left\|\Psi^{(k)}-\mathbf{E}_{\mathbf{0}}\right\| \leq \frac{\rho_{\alpha}}{\left(E_{0}-\alpha\right)}<\left(E_{0}-\alpha\right) \leq \alpha
$$

Therefore, assuming the existence of $\mathcal{R}$, for a given $\alpha$ if $\mathbf{E}^{(k)} \in \mathcal{R}$, then $\Psi^{(k)}$ belongs to an open ball (in $C^{n}$ ) centered in $\mathbf{E}_{\mathbf{0}}$ and radius equal to $\alpha$. Particularly, $\Psi^{(k)} \in \mathcal{R}$ for all $\mathbf{E}^{(0)} \in \mathcal{R}, \forall k$, then if the initial estimate belongs $\mathcal{R}$, all other iterates also belong to $\mathcal{R}$.

\subsection{Convergence Rate}

Once a region where iterates are confided through the iterative process have been deduced, let us examine the convergence rate of the algorithm. Notice that two subsequent iterates of the algorithm can be written as

$$
\begin{aligned}
\psi_{i}^{(k+1)} & =E_{0}-\sum_{m \in \hbar_{i}} \frac{z_{m}}{E_{m}^{(k+1) *}}\left(S_{m, c}^{*}+L_{m, a c}^{(k+1) *}\right) \\
\psi_{i}^{(k)} & =E_{0}-\sum_{m \in \hbar_{i}} \frac{z_{m}}{E_{m}^{(k) *}}\left(S_{m, c}^{*}+L_{m, a c}^{(k) *}\right)
\end{aligned}
$$

and, subtracting (19) from (18) we have

$$
\Delta \psi_{i}^{(k+1)}=\sum_{m \in \hbar_{i}}\left[\frac{z_{m}}{E_{m}^{(k) *}}\left(S_{m, c}^{*}+L_{m, a c}^{(k) *}\right)-\frac{z_{m}}{E_{m}^{(k+1) *}}\left(S_{m, c}^{*}+L_{m, a c}^{(k+1) *}\right)\right]
$$

Hence, by manipulating the terms of the equation above, we have

$$
\Delta \psi_{i}^{(k+1)}=\sum_{m \in \hbar_{i}} z_{m}\left[\frac{S_{m, c}^{*}+L_{m, a c}^{(k+1) *}}{E_{m}^{(k+1) *} E_{m}^{(k) *}}-\frac{\Delta L_{m, a c}^{(k+1) *}}{E_{m}^{(k+1) *} \Delta E_{m}^{(k+1) *}}\right] \Delta E_{m}^{(k+1) *}
$$

where $\Delta E_{m}^{(k+1)}=E_{m}^{(k+1)}-E_{m}^{(k)}$ and $\Delta L_{m, a c}^{(k+1)}=L_{m, a c}^{(k+1)}-L_{m, a c}^{(k)}$.

In a compact form, this expression can be rewritten as

$$
\Delta \psi_{i}^{(k+1)}=\sum_{m \in \hbar_{i}} D_{i m}^{(k+1)} \Delta E_{m}^{(k+1) *}
$$

in which

$$
D_{i m}^{(k+1)}=\sum_{m \in \hbar_{i}} z_{m}\left[\frac{S_{m, c}^{*}+L_{m, a c}^{(k+1) *}}{E_{m}^{(k+1) *} E_{m}^{(k) *}}-\frac{\Delta L_{m, a c}^{(k+1) *}}{E_{m}^{(k+1) *} \Delta E_{m}^{(k+1) *}}\right]
$$

Equation (22) can also be written in matrix notation as

$$
\Delta \Psi^{(k+1)}=\mathbf{D}^{(k+1)} \Delta \mathbf{E}^{(k+1) *}
$$

where $\boldsymbol{\Delta} \boldsymbol{\Psi}^{(k+1)}=\boldsymbol{\Psi}^{(k+1)}-\boldsymbol{\Psi}^{(k)}, \mathbf{D}^{(k+1)}$ is a $n \times n$ matrix with entries given by $D_{i m}^{(k+1)}$ and $\boldsymbol{\Delta} \mathbf{E}^{(k+1)}=$ $\mathbf{E}^{(k+1)}-\mathbf{E}^{(k)}$. Matrix $\mathbf{D}^{(k+1)}$ indicates the convergence rate of the algorithm in each iteration. It also proves convergence direct dependence on network loading, losses iterates and convergence of losses. 


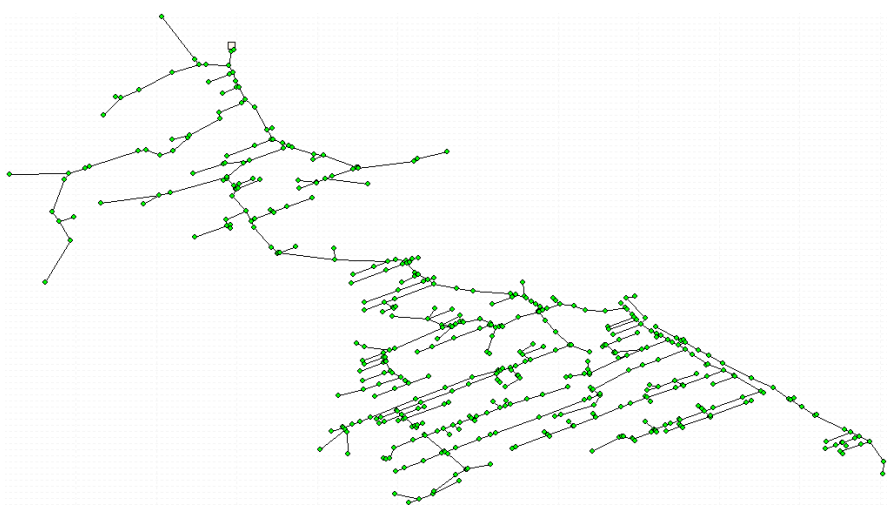

Figure 2. Schematic of the actual distribution network utilized in the evaluations.

\section{NUMERICAL RESULTS}

Numerical load flow analysis are presented in this section to verify the provided mathematical results. Fig. 2 shows an actual $13.80 \mathrm{kV}$ distribution network with 490 nodes utilized to this purpose. Without loss of generality, the value of $8.28 \mathrm{kV}(0.6000 \mathrm{pu})$ has been chosen as $\alpha$. This implies in a feasible region $\mathcal{R} \triangleq$ $\left\{\mathbf{E} \in C^{489},\left\|E_{i}\right\| \geq E_{0}-\alpha, \forall i=1, \ldots, 489\right\}$, which meets the interval

$$
0.5000 \mathrm{pu}=\frac{E_{0}}{2} \leq \alpha<E_{0}-\sqrt{\rho_{\alpha}}=0.8160 \mathrm{pu}
$$

where $\rho_{\alpha}$ equals 0.0339 pu.

Complex voltages have been obtained using the load flow method. Results have been validated using the approach proposed in [1]. Table 1(a) shows the real and imaginary parts of voltage iterates as well as error values for node 300 , assuming the uncommon initial estimate of $2.00 \angle 63.03^{\circ} \mathrm{pu}, \forall i$. On the other hand, Table 1(b) shows the same variables, but for a case considering a loading increased by sevenfold. The maximum absolute mismatch between voltage iterates has been chosen as convergence criterion. The last iterate has been set as solution for the sake of error computation. Tolerance has been specified to $10^{-6}$.

Table 1. Numerical results for bus 300 assuming the initial estimate $E_{i}^{(0)}=2.00 \angle 63.03^{\circ} \mathrm{pu}, \forall i$

(a) Normal loading

\begin{tabular}{ccc||c||c}
\hline$k$ & $e_{300}(\mathrm{pu})$ & $f_{300}(\mathrm{pu})$ & $\mathbf{D}^{(k)}$ & Error \\
\hline 0 & 0.90719 & -1.78241 & - & - \\
1 & 0.99995 & -0.01043 & 0.00013 & $1.79 \mathrm{E}-00$ \\
2 & 0.98115 & -0.00927 & 0.00073 & $2.28 \mathrm{E}-02$ \\
3 & 0.98096 & -0.00936 & 0.00069 & $3.16 \mathrm{E}-04$ \\
4 & 0.98096 & -0.00936 & 0.00079 & $2.91 \mathrm{E}-06$ \\
5 & 0.98096 & -0.00936 & 0.00074 & $2.18 \mathrm{E}-08$ \\
6 & 0.98096 & -0.00936 & 0 & 0 \\
\hline
\end{tabular}

(b) Increased loading

\begin{tabular}{ccc||c|c}
\hline$k$ & $e_{300}(\mathrm{pu})$ & $f_{300}(\mathrm{pu})$ & $\mathbf{D}^{(k)}$ & Error \\
\hline 0 & 0.90719 & -1.78241 & - & - \\
1 & 0.99915 & -0.07408 & 0.00677 & $1.86 \mathrm{E}-00$ \\
2 & 0.85611 & -0.06065 & 0.05374 & $1.94 \mathrm{E}-01$ \\
3 & 0.84061 & -0.06560 & 0.07107 & $2.48 \mathrm{E}-02$ \\
4 & 0.83888 & -0.06560 & 0.07926 & $2.89 \mathrm{E}-03$ \\
5 & 0.83870 & -0.06563 & 0.07756 & $3.13 \mathrm{E}-04$ \\
6 & 0.83868 & -0.06563 & 0.07852 & $3.20 \mathrm{E}-05$ \\
7 & 0.83868 & -0.06563 & 0.07816 & $3.23 \mathrm{E}-06$ \\
8 & 0.83868 & -0.06563 & 0.07826 & $2.96 \mathrm{E}-07$ \\
9 & 0.83868 & -0.06563 & 0 & 0 \\
\hline
\end{tabular}

Fig. 3 shows the first two voltage iterates for the first case in a level curve of the convergence region.

As expected, one can notice that even by choosing a nonrealistic solution as initial estimate, the first iterate belongs to an open ball centered in $E_{0}$ and limited by radius $\alpha$, followed that the convergence of the algorithm is reached. Convergence rate is shown to be, in these cases, lower to the unit. Furthermore, as deduced, the increase of loading caused an increase of convergence rate, impacting on the algorithm efficiency. 


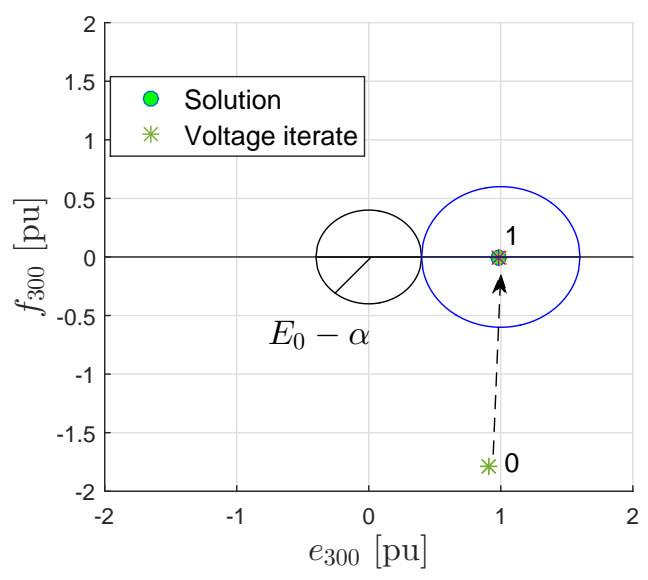

Figure 3. Iterates in a level curve of the region $\mathcal{R}$, for the bus 300 . Initial estimate: $E_{i}^{(0)}=2.00 \angle 63.03^{\circ}$ pu.

\section{CONCLUSIONS}

This paper evaluates the convergence of a load flow method inspired on R. G. Cespedes' recursive equations. Numerical results and formal deduction of convergence rate show that the efficiency of the method depends on the network loading, losses and convergence of losses. Also, a region where algorithm iterates are confined is deduced as long as initial estimates are chosen properly. Future works will extend these developments to three-phase load flow approaches.

\section{ACKNOWLEDGMENTS}

The authors would like to acknowledge the financial, technical and human support of the CNPq, CAPES, INESC Porto and INESC P\&D Brasil.

\section{REFERENCES}

[1] D. Issicaba and J. Coelho, "Rotational load flow method for radial distribution systems," International Journal of Electrical and Computer Engineering (IJEPE), vol. 6, no. 3, 2016.

[2] H. Marefatjou and M. Sarvi, "Distributed generation allocation to improve steady state voltage stability of distribution networks using imperialist competitive algorithm," International Journal of Applied Power Engineering (IJAPE), vol. 2, no. 1, pp. 15-26, 2013.

[3] J. F. Chen and W. M. Wang, "Uniqueness of the feasible voltage solutions for radial power networks," in IEEE Region 10 International Conference on Microeletronics and VLSI, TENCON's 95, Novemeer 1995, pp. 351-354.

[4] H. D. Chiang and M. E. Baran, "On the existence and uniqueness of load flow solution for radial distribution networks," IEEE Transactions on Circuits and Systems, vol. 37, no. 3, pp. 410-416, March 1990.

[5] K. N. Miu and H. D. Chiang, "Existence, uniqueness, and monotonic properties of the feasible power flow solution for radial three-phase distribution networks," IEEE Transactions on Circuits and Systems I:Fundamental Theory and Applications, vol. 47, no. 10, pp. 1502-1514, October 2000.

[6] E. Bompard, E. Carpaneto, G. Chicco, and R. Napoli, "Convergence of the backward-forward sweep method for the load flow analysis of radial distribution systems," Electrical Power \& Energy Systems, vol. 22, no. 7, pp. 521-530, October 2000.

[7] D. Issicaba, "Ladder load flow methods to radial and weakly meshed distribution systems," Master's thesis, Federal University of Santa Catarina, 2008.

[8] R. G. Cespedes, "New method for the analysis of distribution networks," IEEE Transaction on Power Delivery, vol. 5, no. 1, pp. 391-396, January 1990. 


\section{BIOGRAPHIES OF AUTHORS}

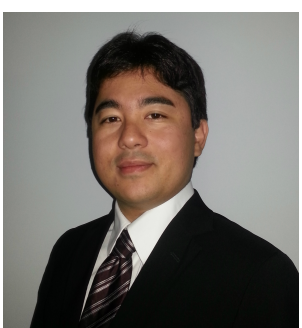

Diego Issicaba received the B.S. and M.S. degrees in Electrical Engineering from the Federal University of Santa Catarina (UFSC), Santa Catarina, Brazil, in 2006 and 2008, respectively. Furthermore, he received the Ph.D. degree on Sustainable Energy Systems, under the MIT Doctoral Program, from the Faculty of Engineering of the University of Porto, Portugal. His research interests involve smart grids, mutiagent systems, distributed generation and distribution systems. He is currently a full Professor at Federal University of Technology - Parana (UTFPR), Associate Researcher and Coordinator of the Research Area on Energy and Management of INESC P\&D Brasil.

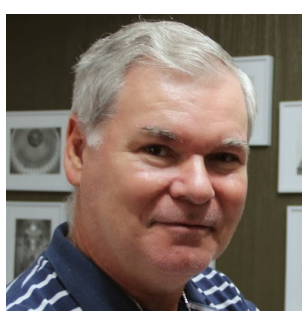

Jorge Coelho received the B.S. and M.S. degrees in electrical engineering from the Federal University of Santa Catarina, Brazil, in 1977 and 1980, respectively. In 1990, he received the Ph.D. degree in electrical engineering from the Catholic University of Rio de Janeiro, Brazil. He is a Professor of the Department of Electrical Engineering at the Federal University of Santa Catarina, Brazil, since March 1978. His research interests include distribution systems expansion and operation planning, power systems reliability, probabilistic methods applied to power systems, and power quality. 